\title{
Lymphomatoid Papulosis Development in Acute Lymphoblastic Leukemia
}

\author{
Kazumi Oura ${ }^{\mathrm{a}}$, Tomonobu Sato ${ }^{\mathrm{a}, \mathrm{d}}\left(\mathbb{0}\right.$, Akihiro Iguchi $^{\mathrm{b}}$, \\ Naohisa Toriumic ${ }^{\mathrm{c}}$, Takeo Sarashina ${ }^{\mathrm{c}}$
}

\begin{abstract}
Lymphomatoid papulosis (LyP) is a chronic, recurrent benign skin disease characterized by histological features of a CD 30-positive cutaneous T-cell lymphoproliferative disorder. It is rare, with an annual, worldwide incidence of $1.2-1.9$ per million, and accounts for $16-47 \%$ of pediatric cutaneous lymphoproliferative disorders. It often occurs on the extremities or the trunk and rarely affects the face or genitals. Its onset may be triggered by irradiation therapy, immunomodulating agents, infection or atopic dermatitis. It has a benign course but is associated with certain hematological malignancies. Mycosis fungoides and primary cutaneous anaplastic large cell lymphoma are the most commonly associated hematological malignancies. The incidence of lymphoma in children with LyP has been reported to be $8.5 \%$ at most. Most patients who develop lymphomas do so within 4 years of the LyP onset; therefore, patients with LyP should be carefully followed up. Herein, we report a case in which tumors appeared in the left scrotum and under the left lip during maintenance therapy for precursor B-cell acute lymphoblastic leukemia. We needed to distinguish the tumor from extramedullary recurrence of ALL or de novo other cutaneous lymphoma; however, the histological findings of a tumor biopsy resulted in a diagnosis of LyP.
\end{abstract}

Keywords: Acute lymphoblastic leukemia; Child; Lymphomatoid papulosis

\section{Introduction}

Lymphomatoid papulosis (LyP) is a chronic and recurrent benign skin disease characterized by histological features of CD30-positive cutaneous T-cell lymphoproliferative disorder.

Manuscript submitted May 21, 2021, accepted May 31, 2021

Published online July 3, 2021

${ }^{a}$ Department of Pediatrics, Kitami Red Cross Hospital, Hokkaido, Japan bepartment of Pediatrics, Hokkaido University Hospital, Hokkaido, Japan 'Department of Pediatrics, Asahikawa Medical University, Hokkaido, Japan ${ }^{\mathrm{d} C}$ Corresponding Author: Tomonobu Sato, Department of Pediatrics, Kitami Red Cross Hospital, North 6-East 2, Kitami 090-8666, Japan.

Email: tomsato1972@gmail.com

doi: https://doi.org/10.14740/jmc3718
LyP is a rare disease with an incidence of $1.2-1.9$ per million [1] and accounts for $16.0-47.0 \%$ of pediatric cutaneous lymphoproliferative disorders [2]. It has been reported that LyP often disappears spontaneously and has a benign course [3, 4]. However, a relationship between LyP and certain hematological malignancies has also been reported [5-7]. Herein, we describe a case in which tumors appeared in the left scrotum and under the left lip during maintenance therapy for precursor B-cell acute lymphoblastic leukemia (BCP-ALL). We needed to distinguish the tumor from extramedullary recurrence of ALL or de novo other cutaneous lymphoma; however, the histological findings of a tumor biopsy resulted in a diagnosis of LyP.

\section{Case Report}

\section{Investigations}

A 2-year-old boy, who had a medical history of mild atopic dermatitis, presented with severe pain in his swollen left knee and prolonged fever. His peripheral white blood cell count was 7,400 cells $/ \mu \mathrm{L}$ with $45 \%$ blast cells, hemoglobin $7.7 \mathrm{~g} / \mathrm{dL}$, and a platelet count $11.4 \times 10^{4} / \mu \mathrm{L}$. Bone marrow examination revealed a massive infiltration of lymphoblasts with chromosome $53, \mathrm{XY},+\mathrm{X},+6,+10,+18,+21,+21,+$ mar, and the leukemic cell surface markers were positive for CD10, CD11a, CD19, CD22, CD34, CD38, HLA-DR, and negative for surface immunoglobulin (Ig). Cerebrospinal fluid examination showed no findings suggestive of central nervous system blast infiltration. Systemic computed tomography (CT) revealed no extramedullary lesions. He was diagnosed with standard risk BCP-ALL. According to the protocol of the ALL-B12 standard risk group of the Japanese Pediatric Leukemia/Lymphoma Study Group, he received intensive chemotherapy with vincristine, L-asparaginase, daunorubicin, prednisolone, and intrathecal injection by methotrexate, hydrocortisone, and cytosine arabinoside to prevent blasts infiltration into central nervous system [8]. Treatment with prednisolone before remission induction therapy rapidly eradicated leukemic cells. Bone marrow findings after induction therapy were complete remission, and chemotherapy of standard risk group was continued. No extramedullary lesions were seen during the treatment course, and his leukemia went into hematological remission and he was followed up as an outpatient. 

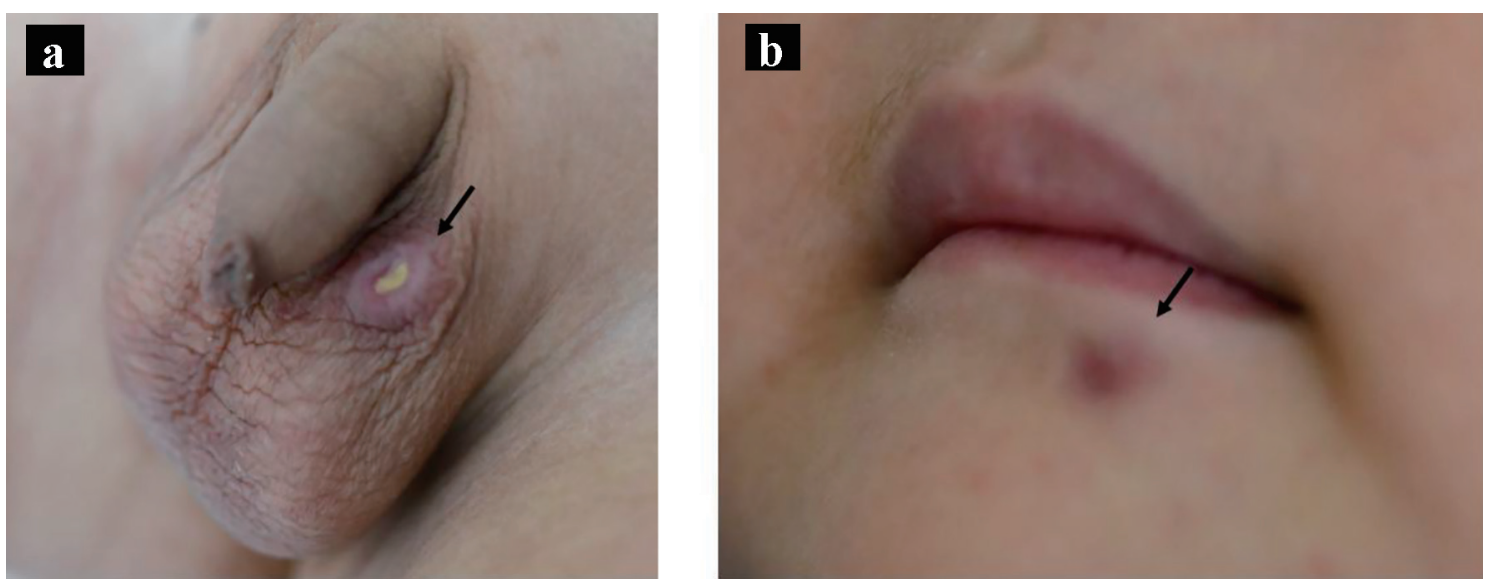

Figure 1. Macroscopic appearance of the tumors on the left scrotum (a, arrow) and under the lip (b, arrow).

\section{Diagnosis}

Seventeen months after starting chemotherapy, during maintenance therapy of daily mercaptopurine hydrate and weekly methotrexate, he developed a firm, dark-red ulcer of $1 \mathrm{~cm}$ diameter in the left scrotum (Fig. 1a) and a tumor $5 \mathrm{~mm}$ long below the left lip (Fig. 1b). No systemic lymphadenopathy or hepatosplenomegaly was observed. He was investigated thoroughly to exclude a leukemic extramedullary relapse. No blast cells were found in the peripheral blood and bone marrow specimens. Laboratory analysis revealed that peripheral white blood cell count was 2,910 cells $/ \mu \mathrm{L}$ with 760 cells $/ \mu \mathrm{L}$ lymphocytes, lactate dehydrogenase was $252 \mathrm{U} / \mathrm{L}$ (normal reference range: 115 - $245 \mathrm{U} / \mathrm{L}$ ) and the serum Ig level was 738 $\mathrm{mg} / \mathrm{dL}$. Systemic CT showed no mass in the testis and no other abnormal findings. The scrotal tumor was biopsied; its histology showed focal necrotic lesions in the dermis and dense cellular infiltration in all dermal layers. The cellular infiltrate mainly consisted of small-to-large mononuclear cells, and immunostaining showed that the large cells were positive for the expression of $\mathrm{CD} 30$. However, there were no sheet-like proliferations or large, atypical cells as seen in typical histological findings of CD30-positive anaplastic large cell lymphoma (Fig. 2a-d). Therefore, these histopathological findings were consistent with a diagnosis of LyP. We expected the tumors to disappear spontaneously, so maintenance chemotherapy was continued. Both tumors subsequently disappeared 2 months later, and the patient was followed at our center for 18 months since the onset of LyP. However, recurrence of neither ALL nor the tumors of LyP was observed.

\section{Discussion}

Since LyP is a rare primary cutaneous CD30-positive lymphoproliferative disorder, it is exceedingly difficult to consider LyP as a differential diagnosis when encountering a mass lesion of the skin, and to diagnose LyP only from its appearance [3]. LyP often occurs on the extremities or trunk, and rarely occurs on the face or genitals [9]. Red or reddish-brown nod- ules generally grow to $1-2 \mathrm{~cm}$ in diameter, and pustules or ulcers may occur on their surface. Differential diagnosis based on the appearance of LyP includes insect bite, eosinophilic granuloma, and pityriasis lichenoides. In addition, in LyP cases with ulcerative lesion, we also need to consider pyoderma gangrenosum and herpesvirus infection [4]. The diagnosis of LyP requires not only pathological findings but also correlation with clinical features and course, and the observation of spontaneous regression of LyP lesions plays a particularly important role. However, in our case, he was undergoing chemotherapy for leukemia and had a tumor nearby the testis where extramedullary relapse frequently occurs in leukemia patients. Therefore, we needed to rule out the extramedullary recurrence of leukemia as soon as possible. Based on the results of the biopsy findings, spontaneous remission of the tumor was expected and he was continued with the planned chemotherapy. Although it is essential to confirm the histological findings for diagnosis of LyP, we should consider when to perform a biopsy based on the individual patient's situation.

Except for the observation that chromosomal rearrangements involving the DUSP22 and IRF4 translocation locus on 6 p25.3 associate with certain subtype of LyP in the elderly [10], there are still many unclear points regarding the etiopahogenesis of LyP. Regarding the spontaneous regression of LyP, Mori et al investigated the interaction between CD30 and its ligand in tumor tissue and reported that spontaneous regressing lesions revealed a significant increase in CD30 ligand expression compared to non-regressive lesions [11]. Moreover, studies report that LyP onset is triggered by irradiation therapy, immunomodulating agents, infection, and atopic dermatitis to date $[4,12,13]$. Our patient certainly had a medical history of mild atopic dermatitis and was treated with immunomodulator agents such as mercaptopurine hydrate and methotrexate for acute leukemia. The details of the correlation between atopic dermatitis and LyP are unknown; however, it has been reported that significant numbers of CD4-positive T lymphocytes express CD30 in inflammatory lesion of patients with atopic dermatitis, and clonal transformation of CD30-positive T cells could cause the development of primary cutaneous CD30-positive lymphoproliferative disorders [14]. Although the incidence of LyP in children is 


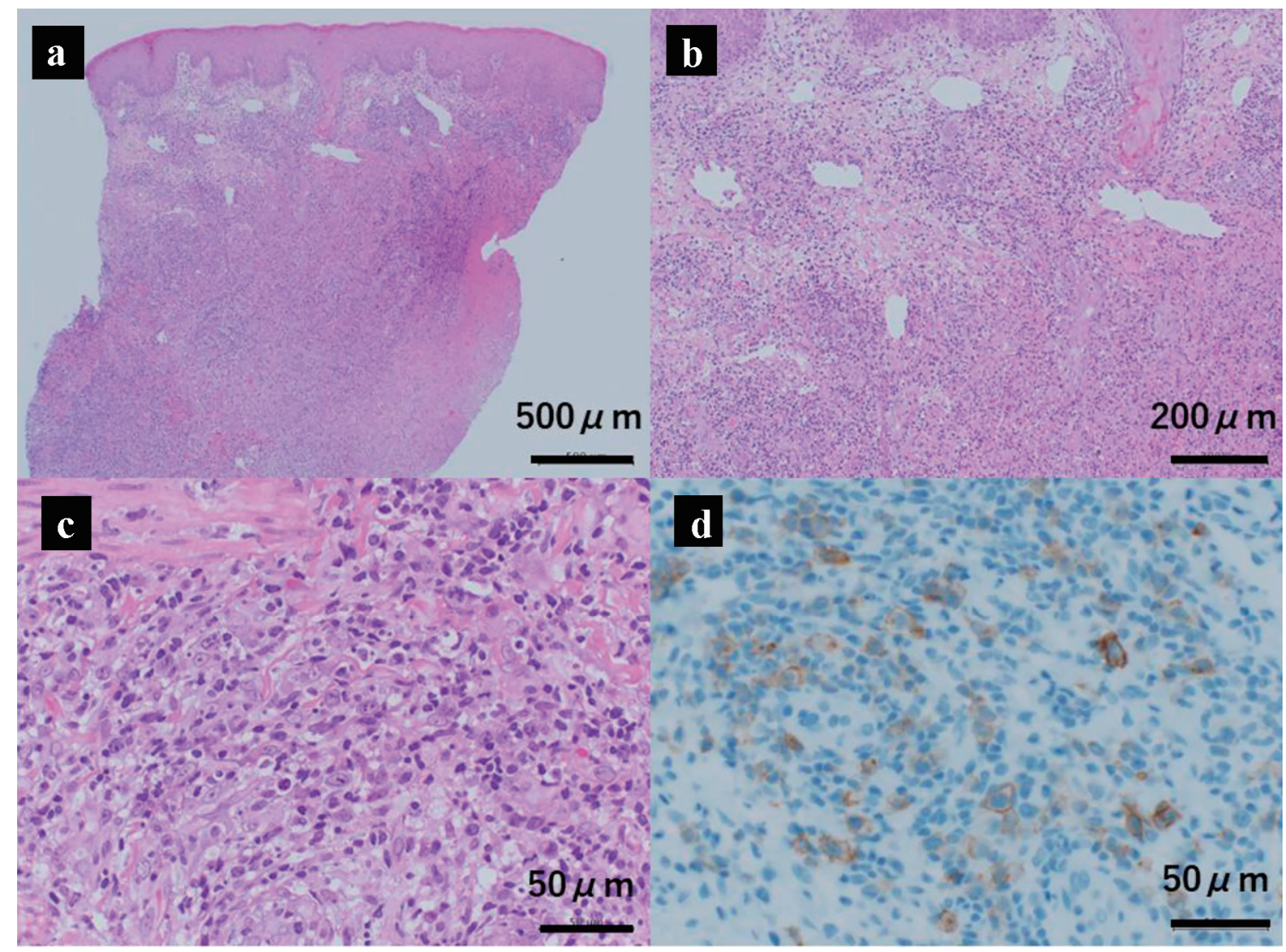

Figure 2. Histology of the scrotal skin mass showing cutaneous CD30-positive T-cell lymphoproliferative disorder (lymphomatoid papulosis). The lower (a) and higher (b, c) magnification views of hematoxylin and eosin stained histological specimens of the scrotal skin mass. There is a necrotic layer in the dermis, and dense cell infiltration throughout the dermis. The infiltrating cells are mainly small to large mononuclear cells. Large cells are positive for CD30 on immunostaining (d).

extremely low, it should be remembered in mind that development of LyP as a cutaneous tumor in patients who are in immunosuppressed state or administered with immunomodulators.

LyP disappears spontaneously in a few weeks; although, it may take months to decades [3]. Therefore, in cases of localized lesion and asymptomatic, we could select options that comprise a "wait-and-see" policy as treatment for LyP $[3,4]$. Because of the biological properties of benign tumors and their high spontaneous remission rate, aggressive treatment for LyP, such as systemic chemotherapy, should be avoided. At best, topical application of corticosteroid and phototherapy are considered. Even in the case of disseminated or multiple lesions, ultraviolet light-based phototherapy or low-dose methotrexate are considered as treatments.

While LyP has a benign course, in rare cases other lymphomas may develop. Mycosis fungoides is the most commonly reported lymphoma associated with LyP, followed by primary cutaneous anaplastic large cell lymphoma $[5,6]$. Due to the risk of developing secondary neoplasms, LyP patients need to be monitored long term so that these potentially lethal LyP-related lymphomas could be detected and treated early. The incidence of LyP-associated lymphomas in children was reported in several literature including systematic review, with a range of $0-8.5 \%$ of pediatric LyP patients developing a sec- ond lymphoma [12, 15-17]. In addition, patients who develop lymphomas do so within 3 - 4 years after the LyP [3]; therefore, careful follow-up should occur. In our case, long-term follow-up of LyP is required along with leukemia.

In conclusion, we must consider LyP in the differential diagnosis of cutaneous tumors found in patients during treatment for hematological malignancies. Although LyP is generally recognized as a benign lymphoproliferative disorder, it can result in repeated spontaneous remission and recurrence. Future reviews of large numbers of LyP cases are essential to elucidate underlying pathophysiological mechanisms and establish the best management approach.

\section{Acknowledgments}

We thank Atsuko Nakazawa, MD, PhD, Department of Clinical Research, Saitama Children's Medical Center, for her support in the pathological diagnosis.

\section{Financial Disclosure}

The authors declare that there is no funding regarding the pub- 
lication of this article.

\section{Conflict of Interest}

The authors indicated no potential conflict of interest.

\section{Informed Consent}

The patient's guardians signed an informed consent to publish this report.

\section{Author Contributions}

$\mathrm{KO}$ and TS wrote the paper; AI, NT, and TS reviewed the paper and gave conceptual advice; all authors read and approved the final manuscript.

\section{Data Availability}

The authors declare that data supporting the findings of this study are available within the article.

\section{Abbreviations}

BCP-ALL: precursor B-cell acute lymphoblastic leukemia; CT: computed tomography; Ig: immunoglobulin; LyP: lymphomatoid papulosis

\section{References}

1. Wang HH, Lach L, Kadin ME. Epidemiology of lymphomatoid papulosis. Cancer. 1992;70(12):2951-2957.

2. Ceppi F, Pope E, Ngan B, Abla O. Primary cutaneous lymphomas in children and adolescents. Pediatr Blood Cancer. 2016;63(11):1886-1894.

3. Wagner G, Rose C, Klapper W, Sachse MM. Lymphomatoid papulosis. J Dtsch Dermatol Ges. 2020;18(3):199205.

4. Kempf W, Kerl K, Mitteldorf C. Cutaneous CD30-positive T-cell lymphoproliferative disorders-clinical and histopathologic features, differential diagnosis, and treatment. Semin Cutan Med Surg. 2018;37(1):24-29.

5. Bekkenk MW, Geelen FA, van Voorst Vader PC, Heule
F, Geerts ML, van Vloten WA, Meijer CJ, et al. Primary and secondary cutaneous $\mathrm{CD} 30(+)$ lymphoproliferative disorders: a report from the Dutch Cutaneous Lymphoma Group on the long-term follow-up data of 219 patients and guidelines for diagnosis and treatment. Blood. 2000;95(12):3653-3661.

6. Wieser I, Oh CW, Talpur R, Duvic M. Lymphomatoid papulosis: Treatment response and associated lymphomas in a study of 180 patients. J Am Acad Dermatol. 2016;74(1):59-67.

7. Kempf W. Cutaneous CD30-Positive Lymphoproliferative Disorders. Surg Pathol Clin. 2014;7(2):203-228.

8. Koh K, Kato M, Saito AM, Kada A, Kawasaki H, Okamoto Y, Imamura T, et al. Phase II/III study in children and adolescents with newly diagnosed B-cell precursor acute lymphoblastic leukemia: protocol for a nationwide multicenter trial in Japan. Jpn J Clin Oncol. 2018;48(7):684691.

9. Willemze R, Meyer CJ, Van Vloten WA, Scheffer E. The clinical and histological spectrum of lymphomatoid papulosis. Br J Dermatol. 1982;107(2):131-144.

10. Karai LJ, Kadin ME, Hsi ED, Sluzevich JC, Ketterling RP, Knudson RA, Feldman AL. Chromosomal rearrangements of 6p25.3 define a new subtype of lymphomatoid papulosis. Am J Surg Pathol. 2013;37(8):1173-1181.

11. Mori M, Manuelli C, Pimpinelli N, Mavilia C, Maggi E, Santucci M, Bianchi B, et al. CD30-CD30 ligand interaction in primary cutaneous $\mathrm{CD} 30(+)$ T-cell lymphomas: A clue to the pathophysiology of clinical regression. Blood. 1999;94(9):3077-3083.

12. Miquel J, Fraitag S, Hamel-Teillac D, Molina T, Brousse $\mathrm{N}$, de Prost $\mathrm{Y}$, Bodemer C. Lymphomatoid papulosis in children: a series of 25 cases. Br J Dermatol. 2014;171(5):1138-1146.

13. Nijsten T, Curiel-Lewandrowski C, Kadin ME. Lymphomatoid papulosis in children: a retrospective cohort study of 35 cases. Arch Dermatol. 2004;140(3):306-312.

14. Zychowska M, Wozniak Z, Maj J. Primary Cutaneous CD30+ Lymphoproliferative disorders in a patient with severe atopic dermatitis: is there a causative link? Acta Derm Venereol. 2018;98(1):123-125.

15. Wieser I, Wohlmuth C, Nunez CA, Duvic M. Lymphomatoid papulosis in children and adolescents: a systematic review. Am J Clin Dermatol. 2016;17(4):319-327.

16. Boccara O, Blanche S, de Prost Y, Brousse N, Bodemer C, Fraitag S. Cutaneous hematologic disorders in children. Pediatr Blood Cancer. 2012;58(2):226-232.

17. Georgesen C, Magro C. Lymphomatoid papulosis in children and adolescents: A clinical and histopathologic retrospective cohort. Ann Diagn Pathol. 2020;46:151486. 